\title{
Numerical Solution of Stiff and Oscillatory Problems using Third Derivative Trigonometrically Fitted Block Method
}

\author{
M. Kida ${ }^{\text {a }}$, S. Adamu ${ }^{\text {b,* }}$, O. O. Aduroja ${ }^{\text {c }}$, T. P. Pantuvo \\ ${ }^{a}$ Department of Mathematics, Modibbo Adama University Yola, Adamawa, Nigeria \\ ${ }^{b}$ Department of Mathematics, Nigerian Army University Biu, Borno, Nigeria \\ ${ }^{c}$ Department of Mathematics, Osun State College of Education Ilesha, Osun, Nigeria \\ ${ }^{d}$ Mathematics and Statistics Department, Federal University Wukari, Taraba, Nigeria
}

\begin{abstract}
This paper considered the formulation of continuous third derivative trigonometrically fitted block method for the solution of stiff and oscillatory problems. The development of the technique involved the interpolation and collocation of the approximate solution which is the combination of polynomial and trigonometric functions. Solving for the unknown parameters and substituting the results into the approximate solution yielded a continuous linear multistep method, which is evaluated at some selected grid points where two cases were considered at equal intervals to give the discrete schemes which are implemented in block form. The blocks are convergent and stable. Numerical experiments show that the methods compete favorably with existing method and efficient for the solution of stiff and oscillatory problems.
\end{abstract}

DOI:10.46481/jnsps.2022.271

Keywords: Third derivative, Trigonometrically fitted block method, Oscillatory problems, Stability, Convergence

Article History :

Received: 22 June 2021

Received in revised form: 27 January 2022

Accepted for publication: 28 January 2022

Published: 28 February 2022

(c)2022 Journal of the Nigerian Society of Physical Sciences. All rights reserved. Communicated by: T. Latunde

\section{Introduction}

In science and engineering, mathematical models are developed to help in the studying of physical phenomena, these models often yield equations that contain some derivatives of an unknown function of one or several variables, such equations are called Differential Equations (DEs) [1, 2]. Interestingly, systems described by differential equations are so complex, or the systems that they describe are so large, that a purely analytical solution to the equations is not tractable, hence the need for numerical approximation [3].

\footnotetext{
${ }^{*}$ Corresponding author tel. no: +2348069405058

Email address: malgwisa@gmail.com (S. Adamu)
}

Block method is formulated in terms of LMM, It preserves the traditional advantage of one step methods, of being selfstarting and permitting easy change of step length $[1,4]$. The advantages of block methods over predictor-corrector methods lies in the fact that they are less expensive in terms of number of functions evaluation, it is capable of giving evaluations at different grid points, without overlapping as done in the predictorcorrector method and it generates simultaneous solutions at all grid points $[1,4,5]$.

Despite the success recorded by linear multistep method (LMM) in the numerical solution of initial value problems, most of the approaches failed when the problems are stiff. Adoption of trigonometrically fitted approximate solution as basis functions have been effective in handling this setback, but their de- 
velopments are tedious. In this paper, we consider numerical procedures for approximating the solution of stiff and oscillatory problems of the form

$$
y^{\prime}=f(x, y,), y\left(x_{0}\right)=\eta_{0} .
$$

These type of differential equations are known to be highly oscillatory and some problems have special properties such as discontinuity and stiffness, hence it is quite difficult to get their numerical solutions accurately $[6,2]$. They are numerically unstable, unless the step size is taken to be extremely small [7]. A stiff system is one involving rapidly changing components together with slowly changing ones, stiff systems are sometimes referred to as systems with large Lipstchitz constant [7, 8]. The system of (1) is stiff if the eigenvalues of the matrix have negative real parts at every time $x$ and varies greatly in magnitude $[9,10]$. Oscillation is the repetitive variation, typically in time, of some measure about a central value (often a point of equilibrium) or between two or more different states [7].

This type of problem arises in different fields of science and engineering, which includes quantum mechanics, celestial mechanics, molecular dynamics, quantum chemistry, astrophysics, electronics and semi-discretizations of wave equation [5, 11].

Numerous numerical methods have been derived for approximating the solutions of (1), some of which are Linear Multistep Methods (LMMs) [12, 13, 4, 14], Block method [5, 15, $1,16,17,18,19,20]$, Trigonometrically fitted methods $[21,22$, $23,6,24]$. Other methods for the solution of Stiff and oscillatory problems includes $[2,9,11]$. Modeled problems in engineering and sciences leads to complex problems such as stiff and oscillatory problems. It was observed that LMM fails to converge at the state of oscillatory as a result has poor stability properties [7]. Second derivative formulas with trigonometric basis functions seems to solve such problems very well and has a better and stability properties $[1,17]$. [25, 26] develop an algorithm for the solution of Stiff Initial Value Problems.

Furthermore, $[27,28]$ considers an implicit $r$-point block backward differentiation formula (BBDF) and block method that generates two values simultaneously respectively for solving stiff IVPs of ODEs. The $r$-point block method simultaneously produces $r$-new values at the time discretization points. The total number of steps to complete the integration by the methods are reduced but not sufficiently and the computation time for the method can still be reduced. [29] developed a block integrator for the solution of stiff and oscillatory first order IVPs of ODEs by means of collocation and interpolation of the combination of power series and exponential function to generate a continuous implicit linear multistep method.

Despite the success achieved by the above methods, it increases the dimension of the resulting systems of first order by the order of the differential equation, hence it wastes both the computer and human effort. Polynomial approximate solution has been reported not to be efficient in handling stiff oscillatory problems, trigonometrically fitted methods handle oscillatory problems effectively, but the application of higher derivatives methods for the solution of first order oscillatory initial value problems has not given much attention.
In this paper Continuous Trigonometrically Fitted Third Derivative Method (CTFTDM) is constructed which provides a discrete method for direct solution of first order initial value problems. The coefficients of the TFTDM are functions of the frequency and the step-size, hence the solutions that will be provided by the methods will be highly accurate if (1) has periodic solutions with the unknown frequencies. This new method considers the application of higher derivative and does not waste both the computer and human effort which is efficient in handling stiff oscillatory problems. Trigonometrically fitted methods are powerful tool in handling stiff/oscillatory problems, order 2 methods developed by combining polynomial and trigonometric approximate solution and considering higher derivatives, conveniently solved stiff/oscillatory problems and it perform better than other methods of the same order.

\subsection{Preliminaries}

Definition 1.1. Stiff [8]: The system

$$
\dot{y}=A y+\phi(x)
$$

where $A$ is an $m \times m$ matrix and $\phi(x)$ an $m$ dimensional vector, is said to be stiff if

$$
\operatorname{Re} \lambda_{i}<0, i=1,2, \ldots, m
$$

and

$$
\frac{\max _{i=1 \ldots m}\left|\operatorname{Re} \lambda_{i}\right|}{\min _{i=1, \ldots, m}\left|\operatorname{Re} \lambda_{i}\right|} \geq 0
$$

where $\lambda_{i}, i=1,2, \ldots, m$ are the eigenvalues of $A$. The ratio

$$
S=\frac{\max _{i=1 \ldots m}\left|\operatorname{Re} \lambda_{i}\right|}{\min _{i=1, \ldots, m}\left|\operatorname{Re} \lambda_{i}\right|} \geq 0
$$

is called the stiffness ratio.

Theorem 1.2. Consistency [30]: A block method is said to be consistent if it has order $p \geq 1$.

Theorem 1.3. Zero-stable [30]: A block method is said to be zero stable if as $h \rightarrow 0$, the roots $r_{j}, j=1(1) k$ of the first characteristic polynomials $\rho(r)=0$ that is

$$
\rho(r)=\operatorname{det}\left[\sum A^{(0)} R^{k-1}\right]=0
$$

satisfying $|R| \leq 1$, must be simple.

Theorem 1.4. Convergent [31]: Consistent and zero stability are sufficient conditions for a block method to be convergent.

\section{Methodology}

We considered the approximate solution

$$
y(x)=\sum_{n=0}^{2} \alpha_{n} x^{n}+\sum_{n=1}^{2} \beta_{n} \sin n \omega x+\sum_{n=1}^{2} c_{n} \cos n \omega x
$$


where $\omega=\frac{2 n \pi}{T}, T$ is the period of the oscillation, $\alpha^{\prime} s, \beta^{\prime} s$ and $c^{\prime} s$ are parameters to be determined.

Interpolating (2) at point $x_{n}$, collocating the first derivative of (2) at point $x_{n}, x_{n+u}, x_{n+v}$, collocating the second derivative of (2) at point $x_{n+u}, x_{n+v}$, and collocating the third derivative of (2) at point $x_{n+v}$ for $u$ and $v, u<v$ to obtain the system

$$
X A=U
$$

where

$$
\begin{gathered}
A=\left[\begin{array}{lccccccc}
\alpha_{1} & \beta_{1} & \beta_{2} & \beta_{3} & c_{1} & c_{2} & d_{2}
\end{array}\right]^{T} \\
X=\left[\begin{array}{cccccccc}
y_{n} & f_{n} & f_{n+u} & f_{n+v} & g_{n+u} & g_{n+v} & l_{n+v}
\end{array}\right]^{T} \\
X=\left[\begin{array}{ccccccc}
1 & x_{n} & x_{n}^{2} & \sin \omega x_{n} & \sin 2 \omega x_{n} & \cos \omega x_{n} & \cos 2 \omega x_{n} \\
0 & 1 & 2 x_{n} & \omega \cos \omega x_{n+v} & 2 \omega \cos 2 \omega x_{n} & -\omega \sin \omega x_{n} & -2 \omega \sin 2 \omega x_{n} \\
0 & 1 & 2 x_{n+u} & \omega \cos \omega x_{n+u} & 2 \omega \cos 2 \omega x_{n+u} & -\omega \sin \omega x_{n+u} & -2 \omega \sin 2 \omega x_{n+u} \\
0 & 1 & 2 x_{n+v} & \omega \cos \omega \omega x_{n+v} & 2 \omega \cos 2 \omega x_{n+v} & -\omega \sin \omega x_{n+v} & -2 \omega \sin 2 \omega x_{n+v} \\
0 & 0 & 2 & -\omega^{2} \sin \omega x_{n+u} & -4 \omega^{2} \sin 2 \omega x_{n+u} & -\omega^{2} \cos \omega x_{n+u} & -4 \omega^{2} \cos 2 \omega x_{n+u} \\
0 & 0 & 2 & -\omega^{2} \sin \omega x_{n+v} & -4 \omega^{2} \sin 2 \omega x_{n+v} & -\omega^{2} \cos \omega x_{n+v} & -4 \omega^{2} \cos 2 \omega x_{n+v} \\
0 & 0 & 0 & -\omega^{3} \cos \omega x_{n+v} & -8 \omega^{3} \cos 2 \omega x_{n+v} & \omega^{3} \sin \omega x_{n+v} & 8 \omega^{3} \sin 2 \omega x_{n+v}
\end{array}\right]
\end{gathered}
$$

(3) gives system of nonlinear equations which is then solved using Newton Raphson's method.

Now considering two cases of third derivative trigonometrically fitted methods at equal intervals as specified below Case I: $u=\frac{1}{2}, v=1$

Case II: $u=1, v=2$

\subsection{Block Method for Case I}

Considering $u=\frac{1}{2}, v=1$

$$
\begin{aligned}
y_{n+t}= & \alpha_{0}(t) y_{n}+\beta_{0}(t) f_{n}+\beta_{1}(t) f_{n+\frac{1}{2}}+\beta_{2}(t) f_{n+1}+\gamma_{n+1}(t) g_{n+\frac{1}{2}} \\
& +\gamma_{n+2}(t) g_{n+2}+\left[l_{n+2}(t)\left(\zeta_{n+2}\right)\right]
\end{aligned}
$$

Evaluating (4) at $t=\frac{1}{2}$ yields

$$
\begin{aligned}
y_{n+\frac{1}{2}}= & \alpha_{01} y_{n}+\beta_{01} f_{n}+\beta_{11} f_{n+\frac{1}{2}}+\beta_{21} f_{n+1}+\gamma_{11} g_{n+\frac{1}{2}} \\
& +\gamma_{21} g_{n+1}+\zeta_{11} l_{n+1}
\end{aligned}
$$

where

$$
\begin{gathered}
\alpha_{01}=1 \\
\beta_{01}=\frac{1}{4} \frac{\left[\begin{array}{c}
8 \cos h \omega+60 \cos 2 h \omega+104 \cos \frac{1}{2} h \omega-94 \cos \frac{3}{2} h \omega-10 \cos \frac{5}{2} h \omega+30 h^{2} \omega^{2} \\
+6 h^{2} \omega^{2} \cos h \omega-33 h^{2} \omega^{2} \cos \frac{1}{2} h \omega-3 h^{2} \omega^{2} \cos \frac{3}{2} h \omega+8 h \omega \sin h \omega \\
+8 h \omega \sin 2 h \omega-56 h \omega \sin \frac{1}{2} h \omega+6 h \omega \sin \frac{3}{2} h \omega-2 h \omega \sin \frac{5}{2} h \omega-68
\end{array}\right]}{\omega\left[\begin{array}{c}
12 \sin h \omega-22 \sin 2 h \omega-42 \sin \frac{1}{2} h \omega+27 \sin \frac{3}{2} h \omega+5 \sin \frac{5}{2} h \omega+26 h \omega \\
+4 h \omega \cos h \omega+2 h \omega \cos 2 h \omega-32 h \omega \cos \frac{1}{2} h \omega+h \omega \cos \frac{3}{2} h \omega-h \omega \cos \frac{5}{2} h \omega
\end{array}\right]} \\
\beta_{11}=\frac{1}{4} \frac{\left[\begin{array}{c}
164 \cos h \omega-44 \cos 2 h \omega-4 \cos 3 h \omega+20 \cos \frac{1}{2} h \omega-42 \cos \frac{3}{2} h \omega \\
+22 \cos \frac{5}{2} h \omega+18 h^{2} \omega^{2}+6 h^{2} \omega^{2} \cos 2 h \omega-30 h^{2} \omega^{2} \cos \frac{1}{2} h \omega \\
+9 h^{2} \omega^{2} \cos \frac{3}{2} h \omega-3 h^{2} \omega^{2} \cos \frac{5}{2} h \omega+32 h \omega \sin h \omega-40 h \omega \sin 2 h \omega \\
+28 h \omega \sin \frac{1}{2} h \omega+6 h \omega \sin \frac{3}{2} h \omega+10 h \omega \sin \frac{5}{2} h \omega-116
\end{array}\right]}{\omega\left[\begin{array}{c}
12 \sin h \omega-22 \sin 2 h \omega-42 \sin \frac{1}{2} h \omega+27 \sin \frac{3}{2} h \omega+5 \sin \frac{5}{2} h \omega+26 h \omega \\
+4 h \omega \cos h \omega+2 h \omega \cos 2 h \omega-32 h \omega \cos \frac{1}{2} h \omega+h \omega \cos \frac{3}{2} h \omega-h \omega \cos \frac{5}{2} h \omega
\end{array}\right]} \\
\beta_{21}=\frac{1}{4} \frac{\left[\begin{array}{c}
2 \\
-172 \cos h \omega-16 \cos 2 h \omega+4 \cos 3 h \omega-124 \cos \frac{1}{2} h \omega+136 \cos \frac{3}{2} h \omega \\
-12 \cos \frac{5}{2} h \omega+4 h^{2} \omega^{2}+2 h^{2} \omega^{2} \cos h \omega-2 h^{2} \omega^{2} \cos 2 h \omega-h^{2} \omega^{2} \cos \frac{1}{2} h \omega \\
-4 h^{2} \omega^{2} \cos \frac{3}{2} h \omega+h^{2} \omega^{2} \cos \frac{5}{2} h \omega-16 h \omega \sin h \omega-12 h \omega \sin 2 h \omega \\
-56 h \omega \sin \frac{1}{2} h \omega+42 h \omega \sin \frac{3}{2} h \omega+2 h \omega \sin \frac{5}{2} h \omega+184
\end{array}\right]}{\omega\left[\begin{array}{c}
12 \sin h \omega-22 \sin 2 h \omega-42 \sin \frac{1}{2} h \omega+27 \sin \frac{3}{2} h \omega+5 \sin \frac{5}{2} h \omega+26 h \omega \\
+4 h \omega \cos h \omega+2 h \omega \cos 2 h \omega-32 h \omega \cos \frac{1}{2} h \omega+h \omega \cos \frac{3}{2} h \omega-h \omega \cos \frac{5}{2} h \omega
\end{array}\right]}
\end{gathered}
$$


$54 \sin h \omega+28 \sin 2 h \omega+6 \sin 3 h \omega+12 \sin \frac{1}{2} h \omega-66 \sin \frac{3}{2} h \omega-14 \sin \frac{5}{2} h \omega$ $-138 h \omega+6 h^{2} \omega^{2} \sin 2 h \omega+18 h^{2} \omega^{2} \sin \frac{1}{2} h \omega-9 h^{2} \omega^{2} \sin \frac{3}{2} h \omega$ $-3 h^{2} \omega^{2} \sin \frac{5}{2} h \omega-6 h \omega \cos h \omega+18 h \omega \cos 2 h \omega-2 h \omega \cos 3 h \omega$

$$
\begin{gathered}
\gamma_{11}=\frac{1}{4} \frac{+156 h \omega \cos \frac{1}{2} h \omega-22 h \omega \cos \frac{3}{2} h \omega-6 h \omega \cos \frac{5}{2} h \omega}{\omega^{2}\left[\begin{array}{c}
12 \sin h \omega-22 \sin 2 h \omega-42 \sin \frac{1}{2} h \omega+27 \sin \frac{3}{2} h \omega+5 \sin \frac{5}{2} h \omega+26 h \omega \\
+4 h \omega \cos h \omega+2 h \omega \cos 2 h \omega-32 h \omega \cos \frac{1}{2} h \omega+h \omega \cos \frac{3}{2} h \omega-h \omega \cos \frac{5}{2} h \omega
\end{array}\right]} \\
\gamma_{21}=\frac{1}{8} \frac{\left[\begin{array}{c}
2 \\
-108 \sin h \omega-56 \sin 2 h \omega-12 \sin 3 h \omega-24 \sin \frac{1}{2} h \omega+132 \sin \frac{3}{2} h \omega \\
+24 h \omega+12 h^{2} \omega^{2} \sin h \omega+30 h^{2} \omega^{2} \sin 2 h \omega+6 h^{2} \omega^{2} \sin \frac{1}{2} h \omega-45 h^{2} \omega^{2} \sin \frac{3}{2} h \omega \\
-3 h^{2} \omega^{2} \sin \frac{5}{2} h \omega+192 h \omega \cos h \omega+40 h \omega \cos 2 h \omega-84 h \omega \cos \frac{1}{2} h \omega \\
+28 \sin \frac{5}{2} h \omega-186 h \omega \cos \frac{3}{2} h \omega+14 h \omega \cos \frac{5}{2} h \omega
\end{array}\right]}{\omega^{2}\left[\begin{array}{c}
12 \sin h \omega-22 \sin 2 h \omega-42 \sin \frac{1}{2} h \omega+27 \sin \frac{3}{2} h \omega+5 \sin \frac{5}{2} h \omega+26 h \omega \\
+4 h \omega \cos h \omega+2 h \omega \cos 2 h \omega-32 h \omega \cos \frac{1}{2} h \omega+h \omega \cos \frac{3}{2} h \omega-h \omega \cos \frac{5}{2} h \omega
\end{array}\right]} \\
\zeta_{11}=\frac{1}{8} \frac{\left[\begin{array}{c}
-156 \cos h \omega-32 \cos 2 h \omega-4 \cos 3 h \omega-144 \cos \frac{1}{2} h \omega+136 \cos \frac{3}{2} h \omega \\
+2 h^{2} \omega^{2}+16 h^{2} \omega^{2} \cos h \omega+14 h^{2} \omega^{2} \cos 2 h \omega-2 h^{2} \omega^{2} \cos \frac{1}{2} h \omega \\
-h^{2} \omega^{2} \cos \frac{5}{2} h \omega-144 h \omega \sin h \omega-24 h \omega \sin 2 h \omega+36 h \omega \sin \frac{1}{2} h \omega \\
+8 \cos \frac{5}{2} h \omega-29 h^{2} \omega^{2} \cos \frac{3}{2} h \omega+126 h \omega \sin \frac{3}{2} h \omega-6 h \omega \sin \frac{5}{2} h \omega+192
\end{array}\right]}{\omega^{3}\left[\begin{array}{c}
12 \sin h \omega-22 \sin 2 h \omega-42 \sin \frac{1}{2} h \omega+27 \sin \frac{3}{2} h \omega+5 \sin \frac{5}{2} h \omega+26 h \omega \\
+4 h \omega \cos h \omega+2 h \omega \cos 2 h \omega-32 h \omega \cos \frac{1}{2} h \omega+h \omega \cos \frac{3}{2} h \omega-h \omega \cos \frac{5}{2} h \omega
\end{array}\right]}
\end{gathered}
$$

Evaluating (4) at $t=1$

$$
\begin{aligned}
y_{n+1}= & \alpha_{02} y_{n}+\beta_{02} f_{n}+\beta_{12} f_{n+\frac{1}{2}}+\beta_{22} f_{n+1}+\gamma_{12} g_{n+\frac{1}{2}} \\
& +\gamma_{22} g_{n+1}+\zeta_{12} l_{n+1}
\end{aligned}
$$

Where

$$
\begin{aligned}
& \alpha_{02}=1 \\
& \beta_{02}=-\frac{1}{2} \frac{\left[\begin{array}{c}
32 \cos h \omega-30 \cos 2 h \omega-34 \cos \frac{1}{2} h \omega+29 \cos \frac{3}{2} h \omega+5 \cos \frac{5}{2} h \omega-20 h^{2} \omega^{2} \\
+18 h^{2} \omega^{2} \cos \frac{1}{2} h \omega+2 h^{2} \omega^{2} \cos \frac{3}{2} h \omega-16 h \omega \sin h \omega-4 h \omega \sin 2 h \omega \\
+70 h \omega \sin \frac{1}{2} h \omega-9 h \omega \sin \frac{3}{2} h \omega+h \omega \sin \frac{5}{2} h \omega-2
\end{array}\right]}{\omega\left[\begin{array}{c}
12 \sin h \omega-22 \sin 2 h \omega-42 \sin \frac{1}{2} h \omega+27 \sin \frac{3}{2} h \omega+5 \sin \frac{5}{2} h \omega+26 h \omega \\
+4 h \omega \cos h \omega+2 h \omega \cos 2 h \omega-32 h \omega \cos \frac{1}{2} h \omega+h \omega \cos \frac{3}{2} h \omega \\
-h \omega \cos \frac{5}{2} h \omega
\end{array}\right]} \\
& \beta_{12}=\frac{\left[\begin{array}{c}
65 \cos h \omega-20 \cos 2 h \omega-\cos 3 h \omega+2 \cos \frac{1}{2} h \omega-9 \cos \frac{3}{2} h \omega \\
+7 \cos \frac{5}{2} h \omega+6 h^{2} \omega^{2}+4 h^{2} \omega^{2} \cos h \omega+2 h^{2} \omega^{2} \cos 2 h \omega \\
+3 h^{2} \omega^{2} \cos \frac{3}{2} h \omega-h^{2} \omega^{2} \cos \frac{5}{2} h \omega+8 h \omega \sin h \omega-16 h \omega \sin 2 h \omega \\
-14 h^{2} \omega^{2} \cos \frac{1}{2} h \omega+28 h \omega \sin \frac{1}{2} h \omega+4 h \omega \sin \frac{5}{2} h \omega-44
\end{array}\right]}{\omega\left[\begin{array}{c}
12 \sin h \omega-22 \sin 2 h \omega-42 \sin \frac{1}{2} h \omega+27 \sin \frac{3}{2} h \omega+5 \sin \frac{5}{2} h \omega+26 h \omega \\
+4 h \omega \cos h \omega+2 h \omega \cos 2 h \omega-32 h \omega \cos \frac{1}{2} h \omega+h \omega \cos \frac{3}{2} h \omega \\
-h \omega \cos \frac{5}{2} h \omega
\end{array}\right]} \\
& \beta_{22}=-\frac{1}{2} \frac{\left[\begin{array}{c}
98 \cos h \omega-10 \cos 2 h \omega-2 \cos 3 h \omega+38 \cos \frac{1}{2} h \omega-47 \cos \frac{3}{2} h \omega \\
+9 \cos \frac{5}{2} h \omega-20 h^{2} \omega^{2}+18 h^{2} \omega^{2} \cos \frac{1}{2} h \omega+2 h^{2} \omega^{2} \cos \frac{3}{2} h \omega \\
+8 h \omega \sin h \omega+16 h \omega \sin 2 h \omega+70 h \omega \sin \frac{1}{2} h \omega-45 h \omega \sin \frac{3}{2} h \omega \\
-3 h \omega \sin \frac{5}{2} h \omega-86
\end{array}\right]}{\omega\left[\begin{array}{c}
12 \sin h \omega-22 \sin 2 h \omega-42 \sin \frac{1}{2} h \omega+27 \sin \frac{3}{2} h \omega+5 \sin \frac{5}{2} h \omega \\
+26 h \omega+4 h \omega \cos h \omega+2 h \omega \cos 2 h \omega-32 h \omega \cos \frac{1}{2} h \omega \\
+h \omega \cos \frac{3}{2} h \omega-h \omega \cos \frac{5}{2} h \omega
\end{array}\right]} \\
& \gamma_{12}=\frac{1}{2} \frac{\left[\begin{array}{c}
39 \sin h \omega-24 \sin 2 h \omega+3 \sin 3 h \omega-12 \sin \frac{1}{2} h \omega-6 \sin \frac{3}{2} h \omega \\
+6 \sin \frac{5}{2} h \omega-26 h \omega+8 h^{2} \omega^{2} \sin h \omega+2 h^{2} \omega^{2} \sin 2 h \omega+4 h^{2} \omega^{2} \sin \frac{1}{2} h \omega \\
-6 h^{2} \omega^{2} \sin \frac{3}{2} h \omega-2 h^{2} \omega^{2} \sin \frac{5}{2} h \omega+17 h \omega \cos h \omega+10 h \omega \cos 2 h \omega \\
-h \omega \cos 3 h \omega+8 h \omega \cos \frac{1}{2} h \omega-8 h \omega \cos \frac{5}{2} h \omega
\end{array}\right]}{\omega^{2}\left[\begin{array}{c}
12 \sin h \omega-22 \sin 2 h \omega-42 \sin \frac{1}{2} h \omega+27 \sin \frac{3}{2} h \omega+5 \sin \frac{5}{2} h \omega \\
+4 h \omega \cos h \omega+2 h \omega \cos 2 h \omega-32 h \omega \cos \frac{1}{2} h \omega+h \omega \cos \frac{3}{2} h \omega \\
+26 h \omega-h \omega \cos \frac{5}{2} h \omega
\end{array}\right]}
\end{aligned}
$$




$$
\begin{aligned}
\gamma_{22}=\frac{1}{2} \frac{\left[\begin{array}{c}
-39 \sin h \omega+24 \sin 2 h \omega-3 \sin 3 h \omega+12 \sin \frac{1}{2} h \omega+6 \sin \frac{3}{2} h \omega \\
-6 \sin \frac{5}{2} h \omega-16 h \omega+4 h^{2} \omega^{2} \sin h \omega+8 h^{2} \omega^{2} \sin 2 h \omega \\
-4 h^{2} \omega^{2} \sin \frac{1}{2} h \omega-12 h^{2} \omega^{2} \sin \frac{3}{2} h \omega+16 h \omega \cos h \omega+28 h \omega \cos \frac{1}{2} h \omega \\
-38 h \omega \cos \frac{3}{2} h \omega+10 h \omega \cos \frac{5}{2} h \omega
\end{array}\right]}{\omega^{2}\left[\begin{array}{c}
12 \sin h \omega-22 \sin 2 h \omega-42 \sin \frac{1}{2} h \omega+27 \sin \frac{3}{2} h \omega+5 \sin \frac{5}{2} h \omega \\
+26 h \omega+4 h \omega \cos h \omega+2 h \omega \cos 2 h \omega-32 h \omega \cos \frac{1}{2} h \omega+h \omega \cos \frac{3}{2} h \omega \\
-h \omega \cos \frac{5}{2} h \omega
\end{array}\right]} \\
\zeta_{12}=\frac{1}{2} \frac{\left[\begin{array}{c}
-63 \cos h \omega+10 \cos 2 h \omega-\cos 3 h \omega-24 \cos \frac{1}{2} h \omega+28 \cos \frac{3}{2} h \omega \\
-4 \cos \frac{5}{2} h \omega+8 h^{2} \omega^{2}+4 h^{2} \omega^{2} \cos h \omega+4 h^{2} \omega^{2} \cos 2 h \omega \\
-8 h^{2} \omega^{2} \cos \frac{1}{2} h \omega-8 h^{2} \omega^{2} \cos \frac{3}{2} h \omega-24 h \omega \sin h \omega-4 h \omega \sin 2 h \omega \\
-24 h \omega \sin \frac{1}{2} h \omega+36 h \sin \frac{3}{2} h \omega-4 h \omega \sin \frac{5}{2} h \omega+54
\end{array}\right]}{\left[\begin{array}{c}
12 \sin h \omega-22 \sin 2 h \omega-42 \sin \frac{1}{2} h \omega+27 \sin \frac{3}{2} h \omega+5 \sin \frac{5}{2} h \omega \\
+26 h \omega+4 h \omega \cos h \omega+2 h \omega \cos 2 h \omega-32 h \omega \cos \frac{1}{2} h \omega \\
+h \omega \cos \frac{3}{2} h \omega-h \omega \cos \frac{5}{2} h \omega
\end{array}\right]}
\end{aligned}
$$

writing (5) and (6) in discrete block method

$$
\begin{aligned}
{\left[\begin{array}{ll}
1 & 0 \\
0 & 1
\end{array}\right]\left[\begin{array}{l}
y_{n+\frac{1}{2}} \\
y_{n+1}
\end{array}\right]=} & {\left[\begin{array}{ll}
0 & 1 \\
0 & 1
\end{array}\right]\left[\begin{array}{c}
y_{n-1} \\
y_{n}
\end{array}\right]+\left[\begin{array}{ll}
0 & \beta_{01} \\
0 & \beta_{02}
\end{array}\right]\left[\begin{array}{c}
f_{n-1} \\
f_{n}
\end{array}\right] } \\
+\left[\begin{array}{ll}
\beta_{11} & \beta_{21} \\
\beta_{12} & \beta_{22}
\end{array}\right]\left[\begin{array}{c}
f_{n+\frac{1}{2}} \\
f_{n+1}
\end{array}\right] & +\left[\begin{array}{ll}
\gamma_{11} & \gamma_{12} \\
\gamma_{21} & \gamma_{22}
\end{array}\right]\left[\begin{array}{c}
g_{n+\frac{1}{2}} \\
g_{n+1}
\end{array}\right]+\left[\begin{array}{ll}
0 & \zeta_{11} \\
0 & \zeta_{21}
\end{array}\right]\left[\begin{array}{l}
l_{n-1} \\
l_{n+1}
\end{array}\right]
\end{aligned}
$$

\subsection{Analysis of the Stability Properties for Case I}

\subsubsection{Order and error constant}

Evaluating each row of (5) and (6) in Taylor series about $x_{n}$ gives

$$
L[y(x) ; h]=y_{n+\frac{1}{2}}-y_{n}-h y_{n}^{\prime}-\beta_{01} f_{n}-\beta_{11} f_{n+\frac{1}{2}}-\beta_{21} f_{n+1}-\gamma_{11} g_{n+\frac{1}{2}}-\gamma_{21} g_{n+1}-\gamma_{11} l_{n+1}=0
$$

therefore, the block methods are of order $p=(2,2)^{T}$ with the following error constants

$$
\begin{aligned}
& -1872 \cos h \omega-384 \cos 2 h \omega-48 \cos 3 h \omega-1728 \cos \frac{1}{2} h \omega+1632 \cos \frac{3}{2} h \omega \\
& +96 \cos \frac{5}{2} h \omega+516 h^{2} \omega^{2}+50 h^{4} \omega^{4}+852 h^{2} \omega^{2} \cos h \omega+540 h^{2} \omega^{2} \cos 2 h \omega \\
& +12 h^{2} \omega^{2} \cos 3 h \omega+16 h^{4} \omega^{4} \cos h \omega-10 h^{4} \omega^{4} \cos 2 h \omega+24 h^{3} \omega^{3} \sin h \omega \\
& +212 h^{3} \omega^{3} \sin 2 h \omega-588 h^{2} \omega^{2} \cos \frac{1}{2} h \omega-1338 h^{2} \omega^{2} \cos \frac{3}{2} h \omega+6 h^{2} \omega^{2} \cos \frac{5}{2} h \omega \\
& -38 h^{4} \omega^{4} \cos \frac{1}{2} h \omega-23 h^{4} \omega^{4} \cos \frac{3}{2} h \omega+5 h^{4} \omega^{4} \cos \frac{5}{2} h \omega-216 h^{3} \omega^{3} \sin \frac{1}{2} h \omega \\
& -180 h^{3} \omega^{3} \sin \frac{3}{2} h \omega-28 h^{3} \omega^{3} \sin \frac{5}{2} h \omega-2376 h \omega \sin h \omega-624 h \omega \sin 2 h \omega \\
& k=-\frac{1}{96} \frac{-72 h \omega \sin 3 h \omega+288 h \omega \sin \frac{1}{2} h \omega+2304 h \omega \sin \frac{3}{2} h \omega+96 h \omega \sin \frac{5}{2} h \omega+2304}{\omega^{3}\left[\begin{array}{c}
12 \sin h \omega-22 \sin 2 h \omega-42 \sin \frac{1}{2} h \omega+27 \sin \frac{3}{2} h \omega+5 \sin \frac{5}{2} h \omega+26 h \omega \\
+4 h \omega \cos h \omega+2 h \omega \cos 2 h \omega-32 h \omega \cos \frac{1}{2} h \omega+h \omega \cos \frac{3}{2} h \omega-h \omega \cos \frac{5}{2} h \omega
\end{array}\right]} \\
& 756 \cos h \omega-120 \cos 2 h \omega+12 \cos 3 h \omega+288 \cos \frac{1}{2} h \omega-336 \cos \frac{3}{2} h \omega+48 \cos \frac{5}{2} h \omega \\
& -132 h^{2} \omega^{2}-34 h^{4} \omega^{4}+51 h^{2} \omega^{2} \cos h \omega-108 h^{2} \omega^{2} \cos 2 h \omega-3 h^{2} \omega^{2} \cos 3 h \omega \\
& +4 h^{4} \omega^{4} \cos h \omega+2 h^{4} \omega^{4} \cos 2 h \omega-24 h^{3} \omega^{3} \sin h \omega-52 h^{3} \omega^{3} \sin 2 h \omega \\
& -66 h^{2} \omega^{2} \cos \frac{1}{2} h \omega+297 h^{2} \omega^{2} \cos \frac{3}{2} h \omega-39 h^{2} \omega^{2} \cos \frac{5}{2} h \omega+22 h^{4} \omega^{4} \cos \frac{1}{2} h \omega \\
& +7 h^{4} \omega^{4} \cos \frac{3}{2} h \omega-h^{4} \omega^{4} \cos \frac{5}{2} h \omega+192 h^{3} \omega^{3} \sin \frac{1}{2} h \omega+18 h^{3} \omega^{3} \sin \frac{3}{2} h \omega \\
& k=\frac{1}{24} \frac{\left[\begin{array}{c}
+2 h^{3} \omega^{3} \sin \frac{5}{2} h \omega+522 h \omega \sin h \omega-96 h \omega \sin 2 h \omega+18 h \omega \sin 3 h \omega+216 h \omega \sin \frac{1}{2} h \omega \\
-468 h \omega \sin \frac{3}{2} h \omega+84 h \omega \sin \frac{5}{2} h \omega-648
\end{array}\right]}{\omega^{3}\left[\begin{array}{c}
12 \sin h \omega-22 \sin 2 h \omega-42 \sin \frac{1}{2} h \omega+27 \sin \frac{3}{2} h \omega+5 \sin \frac{5}{2} h \omega+26 h \omega \\
+4 h \omega \cos h \omega+2 h \omega \cos 2 h \omega-32 h \omega \cos \frac{1}{2} h \omega+h \omega \cos \frac{3}{2} h \omega-h \omega \cos \frac{5}{2} h \omega
\end{array}\right]}
\end{aligned}
$$

2.2.2. Zero stability of the block

$$
\bar{\rho}(\lambda)=\operatorname{det}\left[\lambda A^{(1)}-A^{(0)}\right]=0
$$


where $A^{(0)}, A^{(1)}$ are from (7)

$$
\lambda\left[\begin{array}{ll}
1 & 0 \\
0 & 1
\end{array}\right]-\left[\begin{array}{ll}
0 & 1 \\
0 & 1
\end{array}\right]=\operatorname{det}\left[\begin{array}{cc}
\lambda & -1 \\
0 & \lambda-1
\end{array}\right], \lambda^{2}-\lambda=\lambda(\lambda-1)=0
$$

Thus, solving for $\lambda$

$$
\lambda(\lambda-1)=0
$$

which implies that $\lambda_{1}=0, \lambda_{2}=1$. Hence, using theorem 1, theorem 2, theorem 3, the block method (7) is zero stable and also consistent as its order $p=[2,2]^{T}>1$, thus it is convergent.

\subsubsection{Linear stability}

Applying the test equation $y^{(k)}=\lambda^{(k)} y_{n}$ to yield $y_{n+1}=\mu(z) y_{m}$, $\mu(z)$ is the amplification equation given by

$$
\mu(z)=-\left(A^{(1)}-z \beta^{(1)}-z \gamma^{(1)}-z \zeta^{(1)}\right)^{-1}\left(A^{(0)}+z^{2} \beta^{(0)}+z^{3} \gamma^{(0)}\right)
$$

where $A^{(0)}=\left[\begin{array}{ll}0 & 1 \\ 0 & 1\end{array}\right], \beta^{(0)}=\left[\begin{array}{ll}0 & \beta_{01} \\ 0 & \beta_{02}\end{array}\right], \gamma^{(0)}=\left[\begin{array}{cc}0 & \zeta_{11} \\ 0 & \zeta_{21}\end{array}\right], A^{(1)}=\left[\begin{array}{ll}1 & 0 \\ 0 & 1\end{array}\right], \beta^{(1)}=\left[\begin{array}{ll}\beta_{11} & \beta_{21} \\ \beta_{12} & \beta_{22}\end{array}\right]$ and $\gamma^{(1)}=\left[\begin{array}{ll}\gamma_{11} & \gamma_{12} \\ \gamma_{21} & \gamma_{22}\end{array}\right]$ the matrix $\mu(z)$ has eigenvalues $\left(0,0, \ldots, \xi_{k}\right)$ where $\xi_{k}$ is called the stability function which is the rational function with coefficients.

$$
\mu(z)=\frac{\left[\begin{array}{c}
72 z-234 z^{2} h+9 z h^{3}-102 z^{2} h^{2}+27 z^{2} h^{4}+126 z h \\
-60 h^{3} \omega^{4}-64 h^{4} \omega^{4}+210 z h^{2} \omega^{2}+210 z h^{3} \omega^{2}-108 z h^{4} \omega^{4} \\
-136 z h^{5} \omega^{4}+180 z^{2} h^{3} \omega^{2}+150 z^{2} h^{4} \omega^{2}+36 z^{2} h^{5} \omega^{4}-24 z^{2} h^{6} \omega^{4}
\end{array}\right]}{\left[\begin{array}{c}
72 z-180 z^{2} h+42 z^{3} h^{2}+45 z^{4} h^{3}-60 h^{3} \omega^{4}+210 z h^{2} \omega^{2} \\
-112 z h^{4} \omega^{4}+180 z^{2} h^{3} \omega^{2}-135 z^{3} h^{4} \omega^{2}+42 z^{2} h^{5} \omega^{4}
\end{array}\right]}, 0
$$

\subsubsection{Region of absolute stability}

The region of absolute stability of the block method (7) is shown in Figure 1.

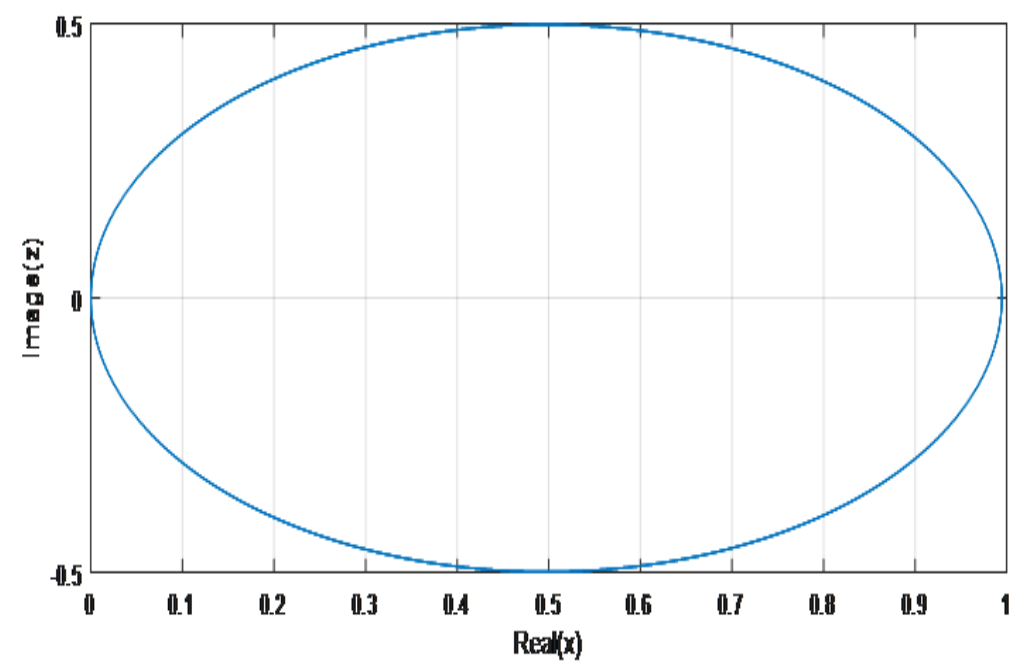

Figure 1. Region of Absolute Stability for Case I

\subsection{Block Method for Case II}

We considered $u=1, v=2$

$$
\begin{aligned}
y_{n+t}= & \alpha_{0}(t) y_{n}+\beta_{0}(t) f_{n}+\beta_{1}(t) f_{n+1}+\beta_{2}(t) f_{n+2}+\gamma_{n+1}(t) g_{n+1} \\
& +\gamma_{n+2}(t) g_{n+2}+\zeta_{n+2}(t) l_{n+2}
\end{aligned}
$$

Evaluating (4) at $t=1$ yields

$$
y_{n+1}=\alpha_{01} y_{n}+\left[\beta_{01} f_{n}+\beta_{11} f_{n+1}+\beta_{21} f_{n+2}\right]+\left[\gamma_{11} g_{n+1}+\gamma_{21} g_{n+2}\right]+\zeta_{11} l_{n+2}
$$


where

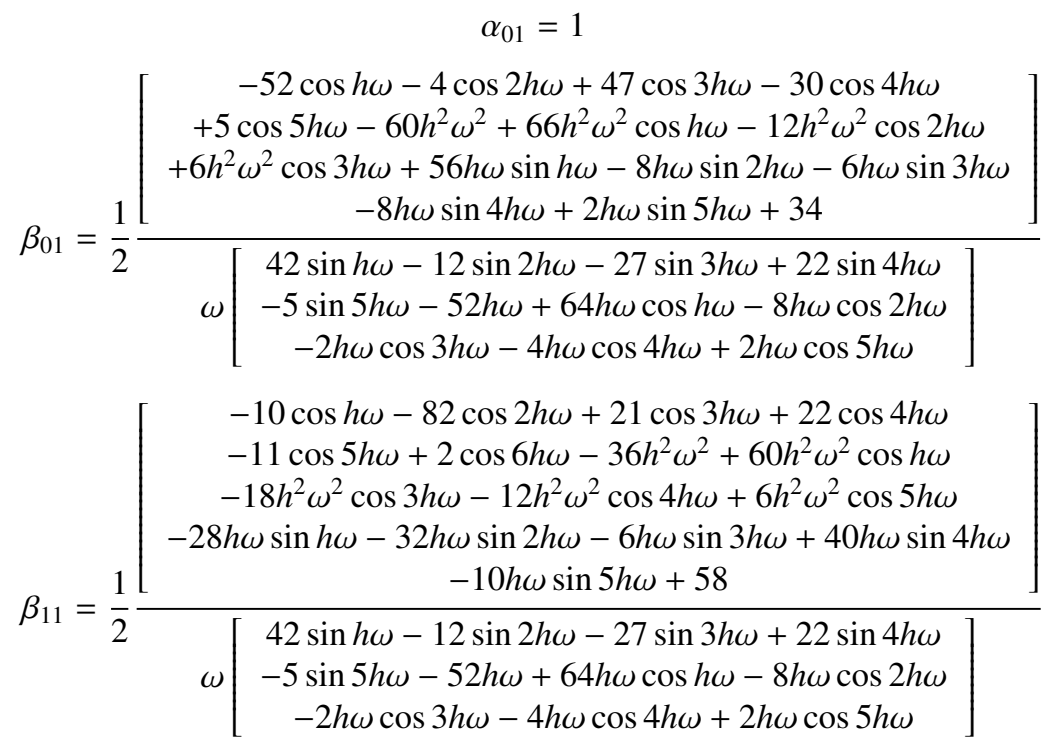
$\beta_{21}=\frac{\left[\begin{array}{c}31 \cos h \omega+43 \cos 2 h \omega-34 \cos 3 h \omega+4 \cos 4 h \omega+3 \cos 5 h \omega \\ -\cos 6 h \omega-4 h^{2} \omega^{2}+h^{2} \omega^{2} \cos h \omega-2 h^{2} \omega^{2} \cos 2 h \omega \\ +4 h^{2} \omega^{2} \cos 3 h \omega+2 h^{2} \omega^{2} \cos 4 h \omega-h^{2} \omega^{2} \cos 5 h \omega \\ +28 h \omega \sin h \omega+8 h \omega \sin 2 h \omega-21 h \omega \sin 3 h \omega \\ +6 h \omega \sin 4 h \omega-h \omega \sin 5 h \omega-46\end{array}\right]}{\omega\left[\begin{array}{c}42 \sin h \omega-12 \sin 2 h \omega-27 \sin 3 h \omega+22 \sin 4 h \omega \\ -5 \sin 5 h \omega-52 h \omega+64 h \omega \cos h \omega-8 h \omega \cos 2 h \omega \\ -2 h \omega \cos 3 h \omega-4 h \omega \cos 4 h \omega+2 h \omega \cos 5 h \omega\end{array}\right]}$ $\gamma_{11}=-\frac{1}{2} \frac{\left[\begin{array}{c}6 \sin h \omega+27 \sin 2 h \omega-33 \sin 3 h \omega+14 \sin 4 h \omega-7 \sin 5 h \omega \\ +3 \sin 6 h \omega-138 h \omega+36 h^{2} \omega^{2} \sin h \omega-18 h^{2} \omega^{2} \sin 3 h \omega \\ +12 h^{2} \omega^{2} \sin 4 h \omega-6 h^{2} \omega^{2} \sin 5 h \omega+156 h \omega \cos h \omega \\ -6 h \omega \cos 2 h \omega-22 h \omega \cos 3 h \omega+18 h \omega \cos 4 h \omega \\ -6 h \omega \cos 5 h \omega-2 h \omega \cos 6 h \omega\end{array}\right]}{\omega^{2}\left[\begin{array}{c}42 \sin h \omega-12 \sin 2 h \omega-27 \sin 3 h \omega+22 \sin 4 h \omega \\ -5 \sin 5 h \omega-52 h \omega+64 h \omega \cos h \omega-8 h \omega \cos 2 h \omega \\ -2 h \omega \cos 3 h \omega-4 h \omega \cos 4 h \omega+2 h \omega \cos 5 h \omega\end{array}\right]}$ $\gamma_{21}=-\frac{1}{2} \frac{\left[\begin{array}{c}-6 \sin h \omega-27 \sin 2 h \omega+33 \sin 3 h \omega-14 \sin 4 h \omega+7 \sin 5 h \omega \\ -3 \sin 6 h \omega+12 h \omega+6 h^{2} \omega^{2} \sin h \omega+12 h^{2} \omega^{2} \sin 2 h \omega \\ -45 h^{2} \omega^{2} \sin 3 h \omega+30 h^{2} \omega^{2} \sin 4 h \omega-3 h^{2} \omega^{2} \sin 5 h \omega-42 h \omega \cos h \omega \\ +96 h \omega \cos 2 h \omega-93 h \omega \cos 3 h \omega+20 h \omega \cos 4 h \omega+7 h \omega \cos 5 h \omega\end{array}\right]}{\omega^{2}\left[\begin{array}{c}42 \sin h \omega-12 \sin 2 h \omega-27 \sin 3 h \omega+22 \sin 4 h \omega \\ -5 \sin 5 h \omega-52 h \omega+64 h \omega \cos h \omega-8 h \omega \cos 2 h \omega \\ -2 h \omega \cos 3 h \omega-4 h \omega \cos 4 h \omega+2 h \omega \cos 5 h \omega\end{array}\right]}$ $\zeta_{11}=\frac{1}{2} \frac{\left[\begin{array}{c}36 \cos h \omega+39 \cos 2 h \omega-34 \cos 3 h \omega+8 \cos 4 h \omega-2 \cos 5 h \omega \\ +\cos 6 h \omega-2 h^{2} \omega^{2}+2 h^{2} \omega^{2} \cos h \omega-16 h^{2} \omega^{2} \cos 2 h \omega \\ +29 h^{2} \omega^{2} \cos 3 h \omega-14 h^{2} \omega^{2} \cos 4 h \omega+h^{2} \omega^{2} \cos 5 h \omega \\ -18 h \omega \sin h \omega+72 h \omega \sin 2 h \omega-63 h \omega \sin 3 h \omega+12 h \omega \sin 4 h \omega \\ +3 h \omega \sin 5 h \omega-48\end{array}\right]}{\omega^{3}\left[\begin{array}{c}42 \sin h \omega-12 \sin 2 h \omega-27 \sin 3 h \omega+22 \sin 4 h \omega \\ -5 \sin 5 h \omega-52 h \omega+64 h \omega \cos h \omega-8 h \omega \cos 2 h \omega \\ -2 h \omega \cos 3 h \omega-4 h \omega \cos 4 h \omega+2 h \omega \cos 5 h \omega\end{array}\right]}$

Evaluating (4) at $t=2$ yields

$$
y_{n+2}=\alpha_{02} y_{n}+\left[\beta_{02} f_{n}+\beta_{12} f_{n+1}+\beta_{22} f_{n+2}\right]+\left[\gamma_{12} g_{n+1}+\gamma_{22} g_{n+2}\right]+\zeta_{12} l_{n+2}
$$


where

$$
\begin{aligned}
& \alpha_{01}=1 \\
& -34 \cos h \omega+32 \cos 2 h \omega+29 \cos 3 h \omega-30 \cos 4 h \omega \\
& +5 \cos 5 h \omega-80 h^{2} \omega^{2}+72 h^{2} \omega^{2} \cos h \omega+8 h^{2} \omega^{2} \cos 3 h \omega \\
& +140 h \omega \sin h \omega-32 h \omega \sin 2 h \omega-18 h \omega \sin 3 h \omega \\
& \beta_{02}=\frac{1}{2} \frac{-8 h \omega \sin 4 h \omega+2 h \omega \sin 5 h \omega-2}{\omega\left[\begin{array}{c}
42 \sin h \omega-12 \sin 2 h \omega-27 \sin 3 h \omega+22 \sin 4 h \omega \\
-5 \sin 5 h \omega-52 h \omega+64 h \omega \cos h \omega-8 h \omega \cos 2 h \omega \\
-2 h \omega \cos 3 h \omega-4 h \omega \cos 4 h \omega+2 h \omega \cos 5 h \omega
\end{array}\right]} \\
& \beta_{12}=\frac{\left[\begin{array}{c}
-2 \cos h \omega-65 \cos 2 h \omega+9 \cos 3 h \omega+20 \cos 4 h \omega \\
-7 \cos 5 h \omega+\cos 6 h \omega-24 h^{2} \omega^{2}+56 h^{2} \omega^{2} \cos h \omega \\
-16 h^{2} \omega^{2} \cos 2 h \omega-12 h^{2} \omega^{2} \cos 3 h \omega-8 h^{2} \omega^{2} \cos 4 h \omega \\
+4 h^{2} \omega^{2} \cos 5 h \omega-56 h \omega \sin h \omega-16 h \omega \sin 2 h \omega \\
+32 h \omega \sin 4 h \omega-8 h \omega \sin 5 h \omega+44
\end{array}\right]}{\omega\left[\begin{array}{c}
42 \sin h \omega-12 \sin 2 h \omega-27 \sin 3 h \omega+22 \sin 4 h \omega \\
-5 \sin 5 h \omega-52 h \omega+64 h \omega \cos h \omega-8 h \omega \cos 2 h \omega \\
-2 h \omega \cos 3 h \omega-4 h \omega \cos 4 h \omega+2 h \omega \cos 5 h \omega
\end{array}\right]} \\
& \beta_{22}=\frac{1}{2} \frac{\left[\begin{array}{c}
38 \cos h \omega+98 \cos 2 h \omega-47 \cos 3 h \omega-10 \cos 4 h \omega \\
+9 \cos 5 h \omega-2 \cos 6 h \omega-80 h^{2} \omega^{2}+72 h^{2} \omega^{2} \cos h \omega \\
+8 h^{2} \omega^{2} \cos 3 h \omega+140 h \omega \sin h \omega+16 h \omega \sin 2 h \omega \\
-90 h \omega \sin 3 h \omega+32 h \omega \sin 4 h \omega-6 h \omega \sin 5 h \omega-86
\end{array}\right]}{\omega\left[\begin{array}{c}
42 \sin h \omega-12 \sin 2 h \omega-27 \sin 3 h \omega+22 \sin 4 h \omega \\
-5 \sin 5 h \omega-52 h \omega+64 h \omega \cos h \omega-8 h \omega \cos 2 h \omega \\
-2 h \omega \cos 3 h \omega-4 h \omega \cos 4 h \omega+2 h \omega \cos 5 h \omega
\end{array}\right]} \\
& \gamma_{12}=-\frac{1}{2} \frac{\left[\begin{array}{c}
-12 \sin h \omega+39 \sin 2 h \omega-6 \sin 3 h \omega-24 \sin 4 h \omega+6 \sin 5 h \omega \\
+3 \sin 6 h \omega-52 h \omega+16 h^{2} \omega^{2} \sin h \omega+32 h^{2} \omega^{2} \sin 2 h \omega \\
-24 h^{2} \omega^{2} \sin 3 h \omega+8 h^{2} \omega^{2} \sin 4 h \omega-8 h^{2} \omega^{2} \sin 5 h \omega \\
+16 h \omega \cos h \omega+34 h \omega \cos 2 h \omega+20 h \omega \cos 4 h \omega \\
-16 h \omega \cos 5 h \omega-2 h \omega \cos 6 h \omega
\end{array}\right]}{\omega^{2}\left[\begin{array}{c}
42 \sin h \omega-12 \sin 2 h \omega-27 \sin 3 h \omega+22 \sin 4 h \omega \\
-5 \sin 5 h \omega-52 h \omega+64 h \omega \cos h \omega-8 h \omega \cos 2 h \omega \\
-2 h \omega \cos 3 h \omega-4 h \omega \cos 4 h \omega+2 h \omega \cos 5 h \omega
\end{array}\right]} \\
& \gamma_{22}=\frac{1}{2} \frac{\left[\begin{array}{c}
-12 \sin h \omega+39 \sin 2 h \omega-6 \sin 3 h \omega-24 \sin 4 h \omega \\
+6 \sin 5 h \omega+3 \sin 6 h \omega+32 h \omega+16 h^{2} \omega^{2} \sin h \omega \\
-16 h^{2} \omega^{2} \sin 2 h \omega+48 h^{2} \omega^{2} \sin 3 h \omega-32 h^{2} \omega^{2} \sin 4 h \omega \\
-56 h \omega \cos h \omega-32 h \omega \cos 2 h \omega+76 h \omega \cos 3 h \omega-20 h \omega \cos 5 h \omega
\end{array}\right]}{\omega^{2}\left[\begin{array}{c}
42 \sin h \omega-12 \sin 2 h \omega-27 \sin 3 h \omega+22 \sin 4 h \omega \\
-5 \sin 5 h \omega-52 h \omega+64 h \omega \cos h \omega-8 h \omega \cos 2 h \omega \\
-2 h \omega \cos 3 h \omega-4 h \omega \cos 4 h \omega+2 h \omega \cos 5 h \omega
\end{array}\right]} \\
& \zeta_{12}=\frac{1}{2} \frac{\left[\begin{array}{c}
24 \cos h \omega+63 \cos 2 h \omega-28 \cos 3 h \omega-10 \cos 4 h \omega \\
+4 \cos 5 h \omega+\cos 6 h \omega-32 h^{2} \omega^{2}+32 h^{2} \omega^{2} \cos h \omega \\
-16 h^{2} \omega^{2} \cos 2 h \omega+32 h^{2} \omega^{2} \cos 3 h \omega-16 h^{2} \omega^{2} \cos 4 h \omega \\
+48 h \omega \sin h \omega+48 h \omega \sin 2 h \omega-72 h \omega \sin 3 h \omega+8 h \omega \sin 4 h \omega \\
+8 h \omega \sin 5 h \omega-54
\end{array}\right]}{\omega^{3}\left[\begin{array}{c}
42 \sin h \omega-12 \sin 2 h \omega-27 \sin 3 h \omega+22 \sin 4 h \omega \\
-5 \sin 5 h \omega-52 h \omega+64 h \omega \cos h \omega-8 h \omega \cos 2 h \omega \\
-2 h \omega \cos 3 h \omega-4 h \omega \cos 4 h \omega+2 h \omega \cos 5 h \omega
\end{array}\right]}
\end{aligned}
$$

writing (11) and (12) in discrete block method

$$
\begin{aligned}
{\left[\begin{array}{ll}
1 & 0 \\
0 & 1
\end{array}\right]\left[\begin{array}{l}
y_{n+1} \\
y_{n+2}
\end{array}\right]=} & {\left[\begin{array}{ll}
0 & 1 \\
0 & 1
\end{array}\right]\left[\begin{array}{c}
y_{n-1} \\
y_{n}
\end{array}\right]+\left[\begin{array}{ll}
0 & \beta_{01} \\
0 & \beta_{02}
\end{array}\right]\left[\begin{array}{c}
f_{n-1} \\
f_{n}
\end{array}\right] } \\
+\left[\begin{array}{ll}
\beta_{11} & \beta_{21} \\
\beta_{12} & \beta_{22}
\end{array}\right]\left[\begin{array}{l}
f_{n+1} \\
f_{n+2}
\end{array}\right] & +\left[\begin{array}{ll}
\gamma_{11} & \gamma_{12} \\
\gamma_{21} & \gamma_{22}
\end{array}\right]\left[\begin{array}{l}
g_{n+1} \\
g_{n+2}
\end{array}\right]+\left[\begin{array}{cc}
0 & \zeta_{11} \\
0 & \zeta_{21}
\end{array}\right]\left[\begin{array}{c}
l_{n-1} \\
l_{n+1}
\end{array}\right]
\end{aligned}
$$


2.4. Analysis of the Stability Properties for Case II

\subsubsection{Order and error constant}

Evaluating each row of (11) and (12) in Taylor series about $x_{n}$ gives

$$
L[y(x) ; h]=y_{n+1}-y_{n}-\beta_{01} f_{n}-\beta_{11} f_{n+1}-\beta_{21} f_{n+2}-\gamma_{11} g_{n+1}-\gamma_{21} g_{n+2}-\zeta_{11} l_{n+2}=0
$$

therefore, the methods are of order $p=(2,2)^{T}$ with the following error constants

$$
\begin{aligned}
& 216 \cos h \omega+234 \cos 2 h \omega-204 \cos 3 h \omega+48 \cos 4 h \omega \\
& -12 \cos 5 h \omega+6 \cos 6 h \omega-258 h^{2} \omega^{2}-100 h^{4} \omega^{4}+294 h^{2} \omega^{2} \cos h \omega \\
& -426 h^{2} \omega^{2} \cos 2 h \omega+669 h^{2} \omega^{2} \cos 3 h \omega-270 h^{2} \omega^{2} \cos 4 h \omega \\
& +76 h^{4} \omega^{4} \cos h \omega-3 h^{2} \omega^{2} \cos 5 h \omega-6 h^{2} \omega^{2} \cos 6 h \omega-32 h^{4} \omega^{4} \cos 2 h \omega \\
& +46 h^{4} \omega^{4} \cos 3 h \omega+20 h^{4} \omega^{4} \cos 4 h \omega-10 h^{4} \omega^{4} \cos 5 h \omega+216 h^{3} \omega^{3} \sin h \omega \\
& -24 h^{3} \omega^{3} \sin 2 h \omega+180 h^{3} \omega^{3} \sin 3 h \omega-212 h^{3} \omega^{3} \sin 4 h \omega+28 h^{3} \omega^{3} \sin 5 h \omega \\
& -72 h \omega \sin h \omega+594 h \omega \sin 2 h \omega-576 h \omega \sin 3 h \omega+156 h \omega \sin 4 h \omega \\
& k=-\frac{1}{12} \frac{-24 h \omega \sin 5 h \omega+18 h \omega \sin 6 h \omega-288}{\omega^{3}\left[\begin{array}{c}
42 \sin h \omega-12 \sin 2 h \omega-27 \sin 3 h \omega+22 \sin 4 h \omega \\
-5 \sin 5 h \omega-52 h \omega+64 h \omega \cos h \omega-8 h \omega \cos 2 h \omega-2 h \omega \cos 3 h \omega \\
-4 h \omega \cos 4 h \omega+2 h \omega \cos 5 h \omega
\end{array}\right]} \\
& k=-\frac{1}{6} \frac{\left.\begin{array}{c}
72 \cos h \omega+189 \cos 2 h \omega-84 \cos 3 h \omega-30 \cos 4 h \omega+12 \cos 5 h \omega \\
+3 \cos 6 h \omega-132 h^{2} \omega^{2}-136 h^{4} \omega^{4}-66 h^{2} \omega^{2} \cos h \omega+51 h^{2} \omega^{2} \cos 2 h \omega \\
+297 h^{2} \omega^{2} \cos 3 h \omega-108 h^{2} \omega^{2} \cos 4 h \omega+88 h^{4} \omega^{4} \cos h \omega-39 h^{2} \omega^{2} \cos 5 h \omega \\
-3 h^{2} \omega^{2} \cos 6 h \omega+16 h^{4} \omega^{4} \cos 2 h \omega+28 h^{4} \omega^{4} \cos 3 h \omega+8 h^{4} \omega^{4} \cos 4 h \omega \\
-4 h^{4} \omega^{4} \cos 5 h \omega+384 h^{3} \omega^{3} \sin h \omega-48 h^{3} \omega^{3} \sin 2 h \omega+36 h^{3} \omega^{3} \sin 3 h \omega \\
-104 h^{3} \omega^{3} \sin 4 h \omega+4 h^{3} \omega^{3} \sin 5 h \omega+108 h \omega \sin h \omega+261 h \omega \sin 2 h \omega \\
-234 h \omega \sin 3 h \omega-48 h \omega \sin 4 h \omega+42 h \omega \sin 5 h \omega+9 h \omega \sin 6 h \omega-162
\end{array}\right]}{\omega^{3}\left[\begin{array}{c}
42 \sin h \omega-12 \sin 2 h \omega-27 \sin 3 h \omega+22 \sin 4 h \omega \\
-5 \sin 5 h \omega-52 h \omega+64 h \omega \cos h \omega-8 h \omega \cos 2 h \omega \\
-2 h \omega \cos 3 h \omega-4 h \omega \cos 4 h \omega+2 h \omega \cos 5 h \omega
\end{array}\right]}
\end{aligned}
$$

\subsubsection{Zero stability of the block}

$$
\bar{\rho}(\lambda)=\operatorname{det}\left[\lambda A^{(1)}-A^{(0)}\right]=0
$$

where $A^{(0)}, A^{(1)}$ are from (13)

$$
\lambda\left[\begin{array}{ll}
1 & 0 \\
0 & 1
\end{array}\right]-\left[\begin{array}{ll}
0 & 1 \\
0 & 1
\end{array}\right]=\operatorname{det}\left[\begin{array}{cc}
\lambda & -1 \\
0 & \lambda-1
\end{array}\right], \lambda^{2}-\lambda=\lambda(\lambda-1)=0
$$

Thus, solving for $\lambda$

$$
\lambda(\lambda-1)=0
$$

which implies that $\lambda_{1}=0, \lambda_{2}=1$ Hence, using theorem 1 , theorem 2 , theorem 3 , the block method (13) is zero stable and also consistent as its order $p=[2,2]^{T}>1$, thus it is convergent.

\subsubsection{Linear stability}

Applying the test equation $y^{(k)}=\lambda^{(k)} y_{n}$ to yield $y_{n+1}=\mu(z) y_{m}$, $\mu(z)$ is the amplification equation given by

$$
\mu(z)=-\left(A^{(1)}-z \beta^{(1)}-z^{2} \gamma^{(1)}-z^{3} \zeta^{(1)}\right)^{-1}\left(A^{(0)}+z \beta^{(0)}+z^{2} \gamma^{(0)}\right)
$$

where $A^{(0)}=\left[\begin{array}{ll}0 & 1 \\ 0 & 1\end{array}\right], \beta^{(0)}=\left[\begin{array}{ll}0 & \beta_{01} \\ 0 & \beta_{02}\end{array}\right], \gamma^{(0)}=\left[\begin{array}{ll}0 & \zeta_{11} \\ 0 & \zeta_{21}\end{array}\right], A^{(1)}=\left[\begin{array}{ll}1 & 0 \\ 0 & 1\end{array}\right], \beta^{(1)}=\left[\begin{array}{ll}\beta_{11} & \beta_{21} \\ \beta_{12} & \beta_{22}\end{array}\right]$ and $\gamma^{(1)}=\left[\begin{array}{ll}\gamma_{11} & \gamma_{12} \\ \gamma_{21} & \gamma_{22}\end{array}\right]$ the matrix $\mu(z)$ has eigenvalues $\left(0,0, \ldots, \xi_{k}\right)$ where $\xi_{k}$ is called the stability function which is the rational function with coefficients.

$$
\mu(z)=\frac{\left[\begin{array}{c}
1364 h^{5} z^{4} \omega^{4}-2816 h^{4} z^{2} \omega^{4}-180 h^{3} z^{5}+280 h^{3} z^{4} \omega^{2} \\
+960 h^{3} \omega^{4}+270 h^{2} z^{3}-420 h^{2} z^{2} \omega^{2}-246 h z^{4}+369 z^{2}
\end{array}\right]}{\left[\begin{array}{c}
-288 h^{5} z^{4} \omega^{4}+288 h^{5} z^{3} \omega^{4}+560 h^{4} z^{2} \omega^{4}-1260 h^{3} z^{4} \omega^{2} \\
+360 h^{3} z^{3} \omega^{2}-960 h^{3} \omega^{4}+150 h^{2} z^{5}+2100 h^{2} z^{2} \omega^{2} \\
+162 h z^{4}-342 h z^{3}-225 z^{2}
\end{array}\right]}, 0
$$


Table 1. Result for Example I

\begin{tabular}{ccccccc}
\hline$h$ & 3BEBDF MAXE & 3BBDF MAXE & Error Case I & Time & Error Case II & Time \\
\hline $10^{-2}$ & $1.68449 \mathrm{e}(-001)$ & $6.62694 \mathrm{e}(-002)$ & $3.6891 \mathrm{e}(-002)$ & $0.178 \mathrm{~s}$ & $2.9440 \mathrm{e}(-002)$ & $0.178 \mathrm{~s}$ \\
$10^{-3}$ & $5.14997 \mathrm{e}(-002)$ & $7.44768 \mathrm{e}(-002)$ & $3.6837 \mathrm{e}(-003)$ & $0.178 \mathrm{~s}$ & $8.3499 \mathrm{e}(-003)$ & $0.178 \mathrm{~s}$ \\
$10^{-4}$ & $6.95725 \mathrm{e}(-003)$ & $8.45376 \mathrm{e}(-003)$ & $2.6596 \mathrm{e}(-004)$ & $0.179 \mathrm{~s}$ & $3.8627 \mathrm{e}(-004)$ & $0.179 \mathrm{~s}$ \\
$10^{-5}$ & $7.16727 \mathrm{e}(-004)$ & $8.53717 \mathrm{e}(-004)$ & $2.6585 \mathrm{e}(-005)$ & $0.179 \mathrm{~s}$ & $1.0017 \mathrm{e}(-005)$ & $0.179 \mathrm{~s}$ \\
\hline
\end{tabular}

\subsubsection{Region of absolute stability}

The region of absolute stability of the block method (13) is shown in Figure 2

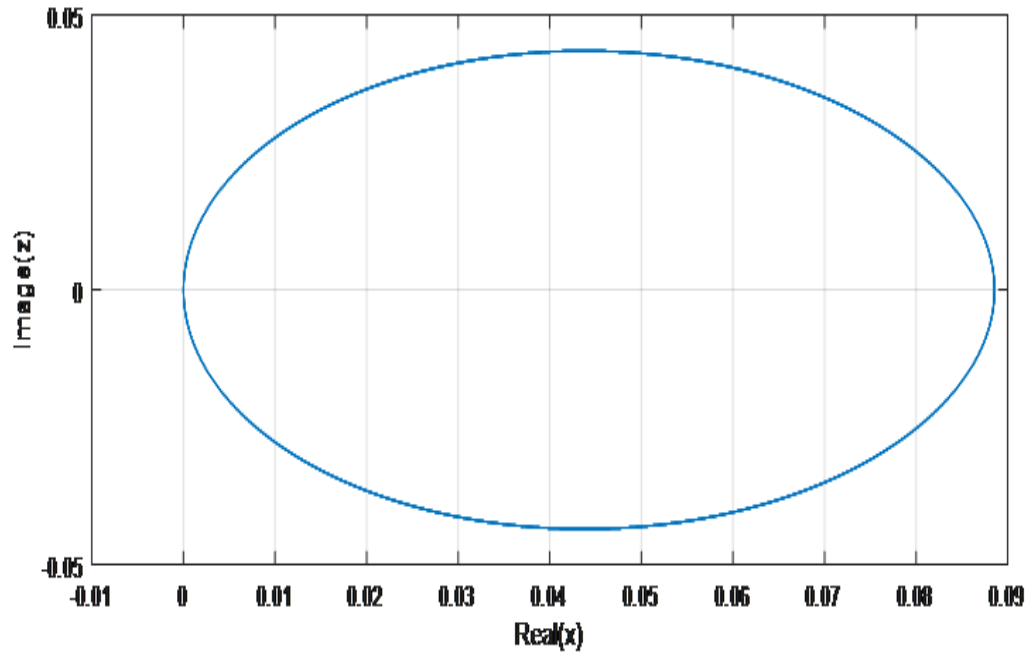

Figure 2. Region of Absolute Stability for Case II

\section{Numerical Experiments}

Evaluating the performance of the new block method on some challenging stiff and oscillatory problems which appeared in literature and compared the results with solution from some methods.

The following notations were used in table:

$h$ Step size

MAXE Maximum Error

$3 B B D F$ 3-point block backward differentiation formula

$3 B E B D F$ 3-point block extended backward differentiation formula

Example I: Consider a linear oscillatory problem in the interval $0 \leq x \leq 10$

$$
\left(\begin{array}{c}
y_{1}^{\prime}=9 y_{1}+24 y_{2}+5 \cos x-\frac{1}{3} \sin x \\
y_{2}^{\prime}=-24 y_{1}-5 y_{2}-9 \cos x+\frac{1}{3} \sin x
\end{array}\right),\left(\begin{array}{l}
y_{1}(0) \\
y_{2}(0)
\end{array}\right)=\left(\begin{array}{c}
\frac{4}{3} \\
\frac{2}{3}
\end{array}\right)
$$

The exact solution is given as

$$
\left(\begin{array}{l}
y_{1}(x) \\
y_{2}(x)
\end{array}\right)=\left(\begin{array}{c}
4 e^{-x}-3 e^{-1000 x} \\
-2 e^{-x}+3 e^{-1000 x}
\end{array}\right)
$$

Source: [25]

Table 1 shows that the new methods performed accurately and approximate better than the result of [25]. For instance, at $h=$ $10^{-5}$, the error in [25] are 7.16727e (-004) and 8.53717e(-004) respectively, while the error in case 1 and case 2 are $2.6585 \mathrm{e}(-05)$ and $1.0017 \mathrm{e}(-05)$ respectively. The comparison of error in Figure 3 clearly shows that, the new methods converge better than the existing method. 


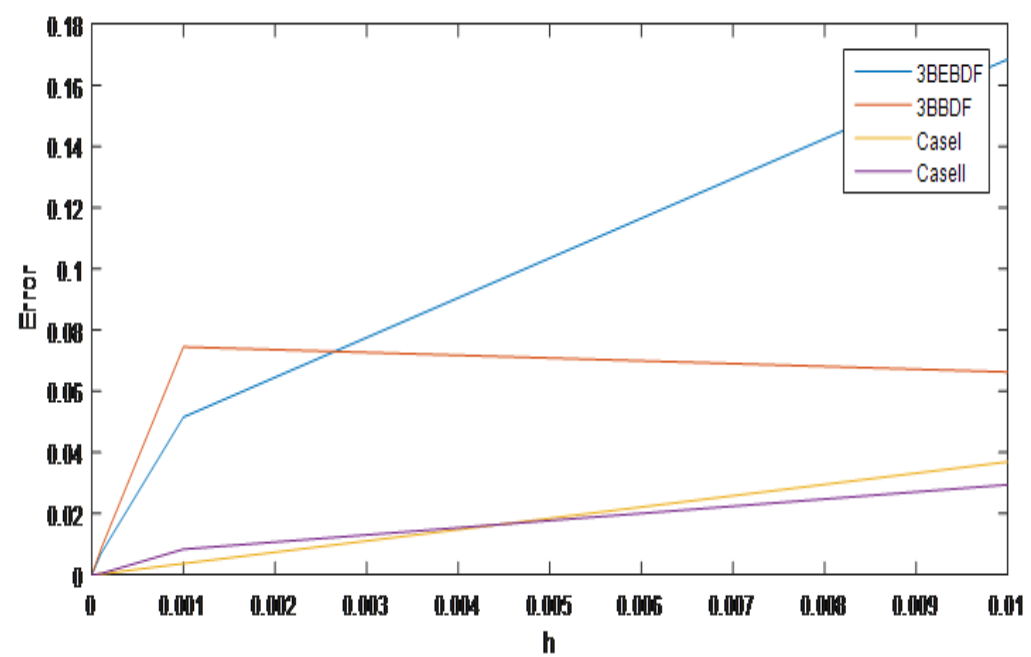

Figure 3. Error for Example I

Table 2. Results for Example II

\begin{tabular}{ccccccc}
\hline$h$ & 3BEBDF MAXE & 3BBDF MAXE & CASE I & Time & CASE II & Time \\
\hline $10^{-2}$ & $8.35359 \mathrm{e}(-002)$ & $1.08041 \mathrm{e}(-002)$ & $1.7729 \mathrm{e}(-05)$ & $0.3511 \mathrm{~s}$ & $1.7729 \mathrm{e}(-05)$ & $0.3511 \mathrm{~s}$ \\
$10^{-3}$ & $9.10218 \mathrm{e}(-003)$ & $1.08962 \mathrm{e}(-002)$ & $1.0051 \mathrm{e}(-04)$ & $0.3511 \mathrm{~s}$ & $2.1635 \mathrm{e}(-06)$ & $0.3511 \mathrm{~s}$ \\
$10^{-4}$ & $9.18073 \mathrm{e}(-004)$ & $1.09230 \mathrm{e}(-003)$ & $5.0175 \mathrm{e}(-05)$ & $0.3511 \mathrm{~s}$ & $2.0646 \mathrm{e}(-07)$ & $0.3511 \mathrm{~s}$ \\
$10^{-5}$ & $9.18862 \mathrm{e}(-005)$ & $1.09257 \mathrm{e}(-004)$ & $4.1886 \mathrm{e}(-06)$ & $0.3510 \mathrm{~s}$ & $7.7533 \mathrm{e}(-08)$ & $0.3510 \mathrm{~s}$ \\
$10^{-6}$ & $9.18939 \mathrm{e}(-006)$ & $1.09259 \mathrm{e}(-005)$ & $4.3258 \mathrm{e}(-07)$ & $0.3510 \mathrm{~s}$ & $7.4327 \mathrm{e}(-09)$ & $0.3510 \mathrm{~s}$ \\
\hline
\end{tabular}

Example II: Consider the linear stiff system in the interval $0 \leq x \leq 10$

$$
\left(\begin{array}{l}
y_{1}^{\prime} \\
y_{2}^{\prime}
\end{array}\right)=\left(\begin{array}{c}
-100 y_{1}+9.901 y_{2} \\
0.1 y_{1}-y_{2}
\end{array}\right),\left(\begin{array}{l}
y_{1}(0) \\
y_{2}(0)
\end{array}\right)=\left(\begin{array}{c}
1 \\
10
\end{array}\right)
$$

The eigenvalues of the Jacobian matrix of the system are $\lambda_{1}=-0.99$ and $\lambda_{2}=-100.01$. The exact solution is given as

$$
\left(\begin{array}{l}
y_{1}(x) \\
y_{2}(x)
\end{array}\right)=\left(\begin{array}{c}
\exp (-0.99 x) \\
10 \exp (-0.99 x)
\end{array}\right)
$$

Source: [25]

Table 2 shows that the new methods performed accurately and approximate better than the result of [25]. At $h=10^{-6}$, the error of [25] are 9.18939e $(-006)$ and $1.09259 \mathrm{e}(-005)$, while the error in case 1 and case 2 are $4.3258 \mathrm{e}(-07)$ and $7.4327 \mathrm{e}(-09)$ respectively. This clearly shows that the new methods performed better than of [25].The comparison of error in Figure 4 clearly shows that, the new methods converge better than the existing method.

Example III: Consider a linear oscillatory problem in the interval $0 \leq x \leq 10$

$$
\left(\begin{array}{l}
y_{1}^{\prime} \\
y_{2}^{\prime}
\end{array}\right)=\left(\begin{array}{c}
-2 y_{1}+y_{2}+2 \sin x \\
998 y_{1}-999 y_{2}+999(\cos x-\sin x)
\end{array}\right),\left(\begin{array}{l}
y_{1}(0) \\
y_{2}(0)
\end{array}\right)=\left(\begin{array}{l}
2 \\
3
\end{array}\right)
$$

The exact solution is given as

$$
\left(\begin{array}{l}
y_{1}(x) \\
y_{2}(x)
\end{array}\right)=\left(\begin{array}{c}
2 \exp (-x)+\sin x \\
2 \exp (-x)+\cos x
\end{array}\right)
$$

Source: [26]

Table 3 shows that the new methods performed accurately and approximate better than the result of [26]. From the result obtained for example III as shown in the Table 3, it is evident that the class of third derivative trigonometrically fitted method 


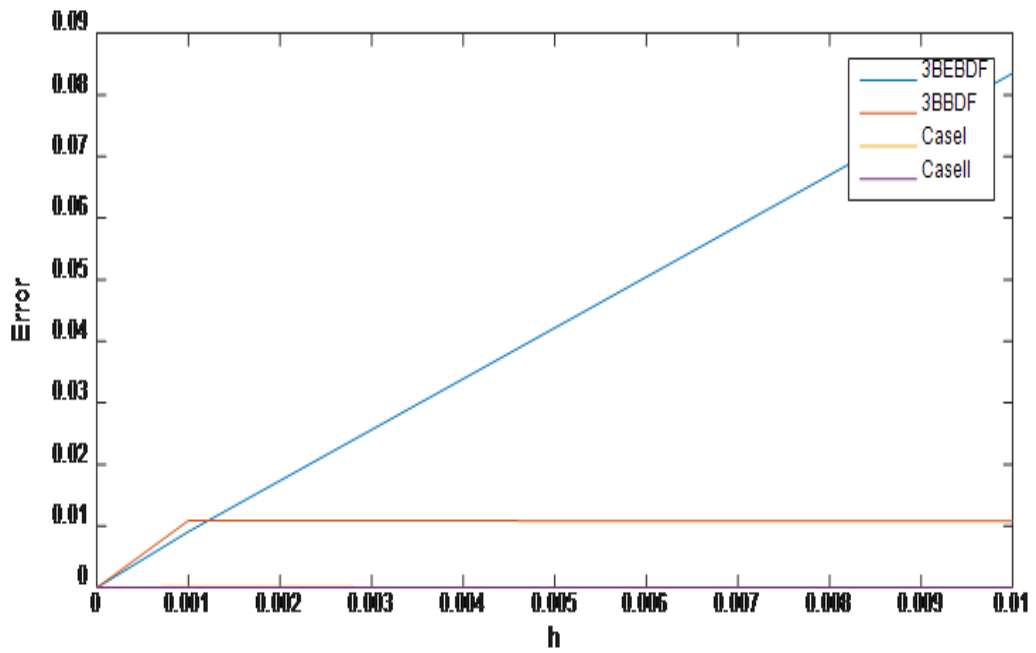

Figure 4. Error for Example II

Table 3. Result for Example III

\begin{tabular}{cccccc}
\hline$h$ & MAXE in [26] & CASE I & Time & CASE II & Time \\
\hline $10^{-2}$ & $8.4000 \mathrm{e}(-003)$ & $5.4393 \mathrm{e}(-04)$ & $0.21322 \mathrm{~s}$ & $2.6947 \mathrm{e}(-04)$ & $0.21322 \mathrm{~s}$ \\
$10^{-4}$ & $1.6621 \mathrm{e}(-004)$ & $2.6947 \mathrm{e}(-05)$ & $0.21322 \mathrm{~s}$ & $1.0276 \mathrm{e}(-06)$ & $0.21322 \mathrm{~s}$ \\
$10^{-6}$ & $2.7506 \mathrm{e}(-006)$ & $1.3195 \mathrm{e}(-08)$ & $0.21321 \mathrm{~s}$ & $6.9756 \mathrm{e}(-08)$ & $0.21321 \mathrm{~s}$ \\
\hline
\end{tabular}

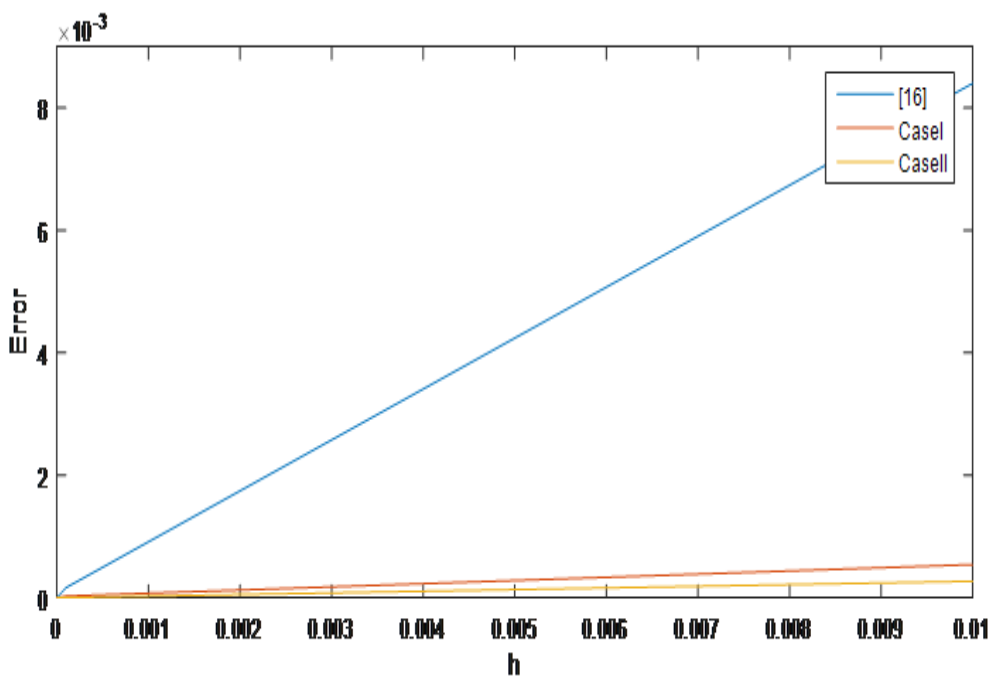

Figure 5. Error for Example III 
Table 4. Result for Example IV

\begin{tabular}{cccc}
\hline Steps & OHBM & CASE I & CASE II \\
\hline 20 & $2.943 \times 10^{-5}$ & $1.311 \times 10^{-5}$ & $1.122 \times 10^{-5}$ \\
40 & $1.250 \times 10^{-6}$ & $1.406 \times 10^{-8}$ & $1.465 \times 10^{-8}$ \\
80 & $2.721 \times 10^{-13}$ & $2.545 \times 10^{-15}$ & $1.843 \times 10^{-15}$ \\
160 & $4.262 \times 10^{-15}$ & $2.733 \times 10^{-18}$ & $1.033 \times 10^{-18}$ \\
\hline 320 & $7.707 \times 10^{-18}$ & $8.371 \times 10^{-20}$ & $9.572 \times 10^{-20}$ \\
\hline 640 & $3.000 \times 10^{-21}$ & $1.721 \times 10^{-21}$ & $1.953 \times 10^{-21}$ \\
\hline Computational Time & $0.03125 \mathrm{~s}$ & $0.02034 \mathrm{~s}$ & $0.02034 \mathrm{~s}$ \\
\hline
\end{tabular}

performed better than those of [26]. The comparison of error in Figure 5 clearly shows that, the new methods converge better than the existing method.

Example IV: Consider the following linear systems

$$
\left(\begin{array}{c}
y_{1}^{\prime}(x)=-21 y_{1}+19 y_{2}-20 y_{3} \\
y_{2}^{\prime}(x)=19 y_{1}-21 y_{2}+20 y_{3} \\
y_{3}^{\prime}(x)=40 y_{1}-40 y_{2}-40 y_{3}
\end{array}\right),\left(\begin{array}{l}
y_{1}(0) \\
y_{2}(0) \\
y_{3}(0)
\end{array}\right)=\left(\begin{array}{c}
1 \\
0 \\
-1
\end{array}\right)
$$

The exact solution is given as

$$
\left(\begin{array}{c}
y_{1}(x) \\
y_{2}(x) \\
y_{3}(x)
\end{array}\right)=\left(\begin{array}{c}
\frac{1}{2}\left(e^{-2 x}+e^{-40 x}(\cos (40 x)+\sin (40 x))\right) \\
\frac{1}{2}\left(e^{-2 x}-e^{-40 x}(\cos (40 x)+\sin (40 x))\right) \\
\frac{1}{2} e^{-40 x}(\cos (40 x)+\sin (40 x))
\end{array}\right)
$$

Source: [2]

We compared the results of CASE I and CASE II along side OHBM by comparing the maximum relative errors over the three components $y_{1}(x), y_{2}(x)$ and $y_{3}(x)$. As shown in Table 4, the new methods in case I and case II proved to be superior in terms of accuracy and computation time.

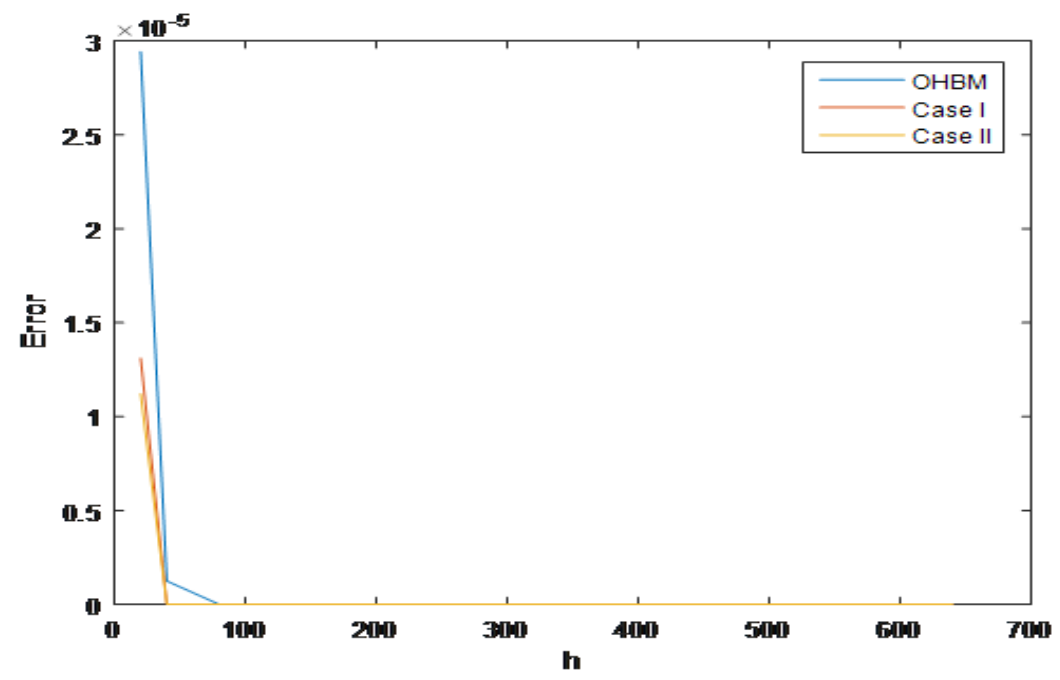

Figure 6. Error for Example IV

Table 5. Result for Example V

\begin{tabular}{cccc}
\hline$h$ & {$[24]$} & ERROR CASE I & ERROR CASE II \\
\hline $2 \pi / 300$ & $2.58313 \times 10^{-9}$ & $2.03334 \times 10^{-9}$ & $2.03034 \times 10^{-9}$ \\
$2 \pi / 600$ & $1.07326 \times 10^{-10}$ & $2.08320 \times 10^{-12}$ & $2.34020 \times 10^{-12}$ \\
$2 \pi / 1200$ & $3.48507 \times 10^{-12}$ & $5.02513 \times 10^{-12}$ & $5.01013 \times 10^{-12}$ \\
\hline
\end{tabular}


Example V: Consider the following problem

$\left(y^{\prime \prime}(x)=-100 y(x)+99 \sin (x)\right), y_{1}(0)=1, y^{\prime}(0)=11, x \in[0,2 \pi]$

The exact solution is given as

$$
y(x)=\cos (10 x)+\sin (10 x)+\sin (x)
$$

Source: [24]

Table 6. Result for Example V

\begin{tabular}{rrrr}
\hline$h$ & {$[24]$} & Error Case I & Error Case \\
\hline $2 \pi / 300$ & $2.58313 \times 10^{-9}$ & $2.03334 \times 10^{-9}$ & $2.03034 \times 10^{-9}$ \\
$2 \pi / 600$ & $1.07326 \times 10^{-10}$ & $2.08320 \times 10^{-12}$ & $2.34020 \times 10^{-12}$ \\
$2 \pi / 1200$ & $3.48507 \times 10^{-12}$ & $5.02513 \times 10^{-12}$ & $5.01013 \times 10^{-12}$ \\
\hline
\end{tabular}

Results of CASE I and CASE II was compared with [24] as shown in Table 5. The new methods compute favorably with [24] and has better consistency.

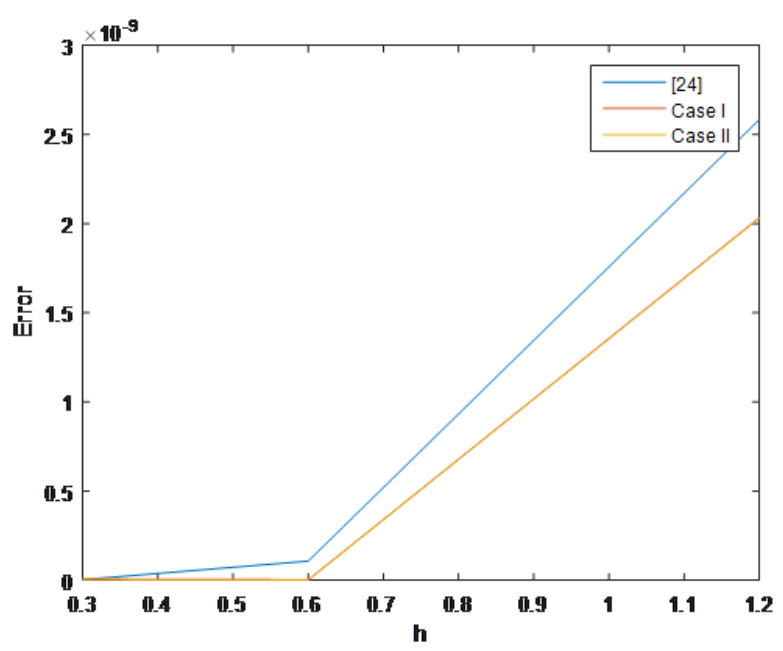

Figure 7. Error for Example V

\section{Conclusion}

A class of third derivative continuous block methods for the solution of stiff and oscillatory problems is constructed using collocation and interpolation technique. The approximate solution adopted for the development of the methods comprises of a combination of polynomial and trigonometric functions. A consistent, zero stable and convergent block methods with continuous coefficients are developed and implemented by writing a code using MATLAB 8.5. The methods are presented in continuous and discrete form.

The accuracy of the block methods were tested on some stiff and oscillatory IVP to generate results and compared the results with the results of some existing methods. The results of the new methods compete favorably with the existing methods with better accuracy, consistency and computational time. Thus the methods are consistent, convergent and zero stable. Hence the methods derived are suitable for solving stiff and oscillatory problems and computationally reliable.

\section{References}

[1] A. O. Adesanya, M. R. Odekunle \& A. O. Adeyeye, "Continuous block hybrid predictor corrector method for the solution of $y^{\prime \prime}=f\left(x, y, y^{\prime}\right)$ ", International Journal of Mathematics and Soft Computing 2 (2020) 35.

[2] M. O. Ogunniran, Y. Haruna, R. B. Adeniyi \& M. O. Olayiwola, "Optimized Three-step Hybrid Block Method for Stiff Problems in Ordinary Differential Equations", Cankaya University Journal of Science and Engineering 17 (2020) 80.

[3] D. Q. Nykamp, "An introduction to ordinary differential equations", From Math Insight. http://mathinsight.org/ordinary_differential_equation_ntroduction $\mathbf{2 0 2 0}$ (2020).

[4] D. O. Awoyemi, S. J. Kayode \& L. O. Adoghe, ”A six-step continuous multistep method for the solution of general fourth order initial value problems of ordinary differential equations", Journal of Natural Sciences Research 5 (2015) 2224.

[5] S. Adamu, A. A. Danhausa, L. Stephen \& B. Williams, "Two hybrid points block methods for the solution of initial value problems", J. of NAMP 54 (2020) 7.

[6] I., F. Fudziah, Z. A. Sufia, D. J. Yusuf \& S. Norazak, "Block Hybrid Method with Trigonometric-Fitting for Solving Oscillatory Problems", Sains Malaysiana 47 (2018) 2223.

[7] V. O. Marcus \& S. Gustaf, "Classification of Stiffness and Oscillations in Initial Value Problems", Masters Thesis, Centre for Mathematical Sciences, Lund University (2015).

[8] R. M. Corless, G. H. Gonnet, D. E. G. Hare, D. J. Jeffrey \& D. E. Knuth, "On the Lambert W Function", Advances in Computational Mathematics 5 (1996) 329.

[9] B. I. Zarina \& S. M. Z. Iskandar, "A Stable Fourth Order Block Backward Differentiation Formulas for Solving Stiff Initial Value Problems", ASM Sci. J. 6 (2019) 60.

[10] B. Bature \& H. Musa, "Stability Analysis of the 2-Point Diagonally Implicit Super Class of Block Backward Differentiation Formula with OffStep Points", J. Phys Math 8 (2017) 1.

[11] N. M. Noor, Z. B. Ibrahim \& F. Ismail, "Numerical solution for stiff initial value problems using 2-point block multistep method", Journal of Physics: Conf. Series 012017 (2019) 1132.

[12] M. O. Udo, R. A. Ademiluyi, A. O. Adesanya \& B. O. Ekpenyong, "An implicit zero stable linear multi-step method for direct solution of general third order initial value problems of ordinary differential equations", Journal of Science and Technology Research 6 (2007) 1596.

[13] E. A. Areo \& R. B. Adeniyi, "A self-starting linear multistep method for direct solution of initial value problems of second order ordinary differential equations", International Journal of Pure and Applied Mathematics 8 (2013) 345.

[14] A. K. Ezzeddine \& G. Hojjati, "Third derivative multistep methods for stiff systems", International Journal of Nonlinear Science 14 (2012) 443.

[15] J. M. Franco, "A class of explicit two-step hybrid methods for secondorder IVPs", Journal of Computational and Applied Mathematics 187 (2006) 41.

[16] T. A. Anake, D. O. Awoyemi \& A. O. Adesanya, "One-step implicit hybrid block method for the direct solution of general second order ordinary differential equations", IAENG International Journal of Applied Mathematics 42 (2012).

[17] A. O. Adesanya, M. R. Odekunle \& M. A. Alkali, "Three steps block predictor corrector method for the solution of general second order ordinary differential equations", Engineering Research and Applications 2 (2012) 2297.

[18] F. F. Ngwane \& S. N. Jator, "Block hybrid-second derivative method for stiff systems", International Journal of Pure and Applied Mathematics 80 (2012) 543.

[19] R. A. Ademiluyi, M. K. Duromola \& B. Bolarinwa, ”Modified block method for the direct solution of initial value problems of fourth order ordinary differential equations", Australian Journal of Basic and Applied Sciences 8 (2014) 389.

[20] K. M. Abualnaja, "A block procedure with linear multi-step methods using Legendre polynomials for solving ODEs", Applied Mathematics 6 (2015) 717.

[21] F. F. Ngwane \& S. N. Jator, "Solving oscillatory problems using a block hybrid trigonometrically fitted method with two off-step points", Ninth MSU-UAB Conference on Differential Equations and Computational 
Simulations. Electronic Journal of Differential Equations Conference 20 (2013) 119

[22] P. L. Ndukum, T. A. Biala, S. N. Jator \& R. B. Adeniyi, ”A fourth order trigonometrically fitted method with the block unification implementation approach for oscillatory initial value problems", International Journal of Pure and Applied Mathematics 103 (2015) 201.

[23] F. F. Ngwane \& S. N. Jator, "A family of trigonometrically fitted enright second derivative methods for stiff and oscillatory initial value problems", Journal of Applied Mathematics 1 (2015).

[24] H. Ramos. Z. Kalogiratou, Th. Monovasilis \& T. E. Simos, ”A Trigonometrically Fitted Optimized Two-step Hybrid Block Method for Solving Initial Value Problems of the form $y^{\prime \prime}=f(x, y, y \prime)$ with Oscillatory Solutions", AIP Conference Proceedings 1 (2015) 1648.

[25] H. Musa, M. B. Suleiman \& N. Senu, "Fully Implicit 3-Point Block Extended Backward Differentiation Formula for Stiff Initial Value Problems", Applied Maths Sciences 6 (2012) 4211.

[26] S. A. M. Yatim, Z. B. Ibrahim, K. I. Othman \& M. B. Suleiman, "A Numerical Algorithm for Solving Stiff Ordinary Differential Equations", Hindawi Publishing Corporation Mathematical Problems in Engineering
11 (2013).

[27] H. Musa, M. B. Suleiman, F. Ismail, N. Senu \& Z. B. Ibrahim, ”An Improved 2-point Block Backward Differentiation Formula for Solving Stiff Initial Value Problems", AIP Conference Proceedings 211 (2013) 1522.

[28] J. Sunday, M. R. Odekunle, A. A. James \& A. O. Adesanya, "Numerical Solution of Stiff and Oscillatory Differential Equations Using a Block Integrator", British Journal of Mathematics and Computer Science 4 (2014) 2471.

[29] Z. B. Ibrahim, K. Othman \& M. Suleiman, "Implicit r-point block Backward Differentiation Formula for Solving First-order Stiff ODEs", Applied Mathematics and Computation 186 (2007) 558.

[30] A. O. Adesanya, B. Abdulqadri \& Y. S. Ibrahim, "Hybrid one step block method for the solution of third order initial value problems of ordinary differential equations", International Journal of Applied Mathematics and Computation 6 (2014) 10.

[31] R. Abdelrahim \& Z. Omar, "A four-step implicit block method with three generalized off-step points for solving fourth order initial value problem directly”, Journal of King Saud University Science 29 (2017) 401. 